\title{
Short-Pulse Laser-Driven Moderated Neutron Source
}

\author{
Sven C. Vogel ${ }^{1, *}$, Juan C. Fernandez ${ }^{1}$, D. Cort Gautier ${ }^{1}$, Nikodem Mitura $^{2}$, Markus Roth $^{2}$, and Kurt F. Schoenberg ${ }^{1,2}$ \\ ${ }^{1}$ Los Alamos National Laboratory, Los Alamos, NM 87545, U.S.A. \\ ${ }^{2}$ TU Darmstadt, D- 64289 Darmstadt, Germany
}

\begin{abstract}
Neutron production with laser-driven neutron sources was demonstrated. We outline the basics of laser-driven neutron sources, highlight some fundamental advantages, and quantitatively compare the neutron production at the TRIDENT laser sources with the well-established LANSCE pulsed neutron spallation source. Ongoing efforts by our team to continue development of these sources, in particular the LANSCE-ina-box instrument, are described. The promise of ultra-intense lasers as drivers for brilliant, compact, and highly efficient particle accelerators portends driving next-generation neutron sources, potentially replacing in some cases much larger conventional accelerators.
\end{abstract}

\section{Introduction}

Neutrons play an important role in material characterization. Neutron based characterization techniques include

- neutron radiography, covering cold, thermal, epithermal, and fast neutron radiography,

- neutron diffraction,

- inelastic neutron scattering,

- neutron reflectometry,

- small angle neutron scattering, and

- neutron cross-section measurements covering all neutron energies

For about half a century, research reactors enabled these applications. Major research reactor facilities include the Institute Laue-Langevin (ILL) in Grenoble, France, or the High Flux Isotope Reactor (HFIR) in Oak Ridge, USA. Reactors, however, have fundamental source brightness limitations that are set by constraints on reactor power density. Accelerator-driven sources on the other hand can surpass the intrinsic source brightness limitations of reactors and avoid the proliferation concerns and nuclear waste concerns arising from the use of highly enriched uranium, which often provides the fuel for these reactor sources..

Since the 1970s, spallation neutron sources became more and more prominent. Spallation neutron sources are driven by high-intensity proton beams and can achieve a source brightness that exceeds the performance of reactors. The most recent major research neutron sources, such as

- the Spallation Neutron Source (SNS) in Oak Ridge, TN, USA,

- the Japan Proton Accelerator Research Complex (J-PARC) at Tokai, Japan, or

- the Chinese Spallation Neutron Source (CSNS) at Dongguan, China, are all spallation sources. Similarly, the planned European Spallation Source (ESS) in Lund, Sweden, will be a spallation neutron source.

The total facility cost for a high-power spallation neutron source exceeds \$1B. Hence, there are few of these sources world-wide. This cost factor, along with the concurrent closure of many smaller research reactors, in particular in Europe, have decreased the ensemble of neutron sources available for research. The Union for Compact Accelerator-driven Neutron Sources (UCANS) has recognized this problem, pointing out that a reduction in available neutron sources will also adversely impact training of the next generation of neutron users at the larger facilities and ultimately jeopardizes neutron research as whole. This concern has driven projects, such as the Jülich high-brilliance neutron source project [1], which will pave the way for research neutron sources at a lower investment cost and with greater accessibility to the research community.

While neutron research plays an important role in the academic or scientific research communities, neutronbased sources for commercial (e.g. neutron radiography for quality control) or government applications (e.g. detection of nuclear contraband) are actively under development and commercially available. Typically, D-D or D-T neutron generators are utilized for these applications with attendant limitations such as the need to use tritium to reach $\sim 10^{13} \mathrm{n} / \mathrm{s}$ versus the $\sim 10^{12} \mathrm{n} / \mathrm{s}$ available with D-D sources. However, all acceleratordriven sources are ultimately flux-limited by chargedensity considerations. However, most applications would greatly benefit from more intense sources, which could also enable entirely new applications such as neutron absorption resonance based characterizations of fission products in nuclear fuels [2] or bulk temperature measurements $[3,4]$, at similar investment cost.

\footnotetext{
* Corresponding author: sven@lanl.gov
} 
In this paper, we describe an alternative approach for the generation of short, intense neutron pulses based on highpower, short-pulse lasers. We describe the principle of this technique and lay out the advantages of laser-based neutron generation compared with conventional RF accelerator-based sources. We quantitatively compare the laser-driven, short-pulse neutron source with the spallation neutron source at the Los Alamos LANSCE facility. Examples of current applied research, as well as the prospects of such novel laser-driven compact neutron sources based on available or proposed laser systems, are discussed.

The promise of ultra-intense lasers as drivers for brilliant, compact, and highly efficient particle accelerators for electrons and ions (potentially replacing in some cases much larger conventional accelerators) also portends driving next-generation neutron sources. Laser-driven sources, at present, cannot compete with acceleratordriven sources or high flux reactors in terms of average neutron flux (where average flux is the number of particles per second regardless of temporal substructure). However, for medium size neutron sources as well as for high peak flux neutron sources, the performance of laser sources is evolving to where they are competitive.

\section{Principles of Laser-Driven Neutron Sources}

Neutrons are produced by nuclear reactions. These reactions can be driven by the interaction of electromagnetic radiation, energetic electrons, or ions with atomic nuclei. For example, a high-velocity ion can break up in the Coulomb field of another atom releasing one or more neutrons. In addition, neutrons can be released by fission reactions, fusion reactions, or by proton-induced spallation of heavy target nuclei.

Los Alamos National Laboratory (LANL) and collaborators have pioneered a novel, short duration yet extremely intense neutron source using the short-pulse laser at LANL's TRIDENT laser facility [5-9]. The TRIDENT laser facility was, until it's closure in 2016, one of the most intense and powerful short-pulse lasers in the world, providing a very high contrast laser pulse of $\sim 600 \mathrm{fs}$ in duration, $\sim 80 \mathrm{~J}$ energy, and with a wavelength of $1053 \mathrm{~nm}$. The laser beam is concentrated to a peak intensity up to $10^{21} \mathrm{~W} / \mathrm{cm}^{2}$. That intensity, when interacting with a sub-micron ultrathin $\mathrm{CD}$ (or $\mathrm{CD}_{2}$ ) foil target, drives a high-energy (peaked around 15-30 MeV, see [5] for details) deuteron beam. The intense deuteron beam then interacts with a beryllium target ("catcher") to produce neutrons.

The TRIDENT laser-driven neutron source featured high intensity and directionality, with approximately $10^{10}$ fast neutrons per shot in a $\sim 1$ steradian cone and with an extremely short neutron pulse of $<1$ ns duration $[5,6]$. The source has been used to develop a new generation of active interrogation concepts to detect clandestine nuclear material [9]. In order to enable other applications, such as nuclear resonance spectroscopy for isotopic identification of (irradiated) nuclear fuel [10], temperature measurement in shock-driven dynamic material experiments $[3,4]$, and pulsed neutron diffraction and scattering, we developed a bright, moderated short-pulse laser-driven neutron source [6]. Beryllium and highdensity polyethylene were used to achieve this aim. The single pulse, moderated neutron source was fully characterized with respect to its energy spectral components by a set of neutron time-of-flight detectors. The energy analysis included both the fast neutron component ( $\sim 1 \mathrm{MeV}$ to $\sim 50 \mathrm{MeV}$, see [5] for details) coming from the neutron production, as well as the epithermal, thermal, and cold (few meV) components from the neutron moderation.

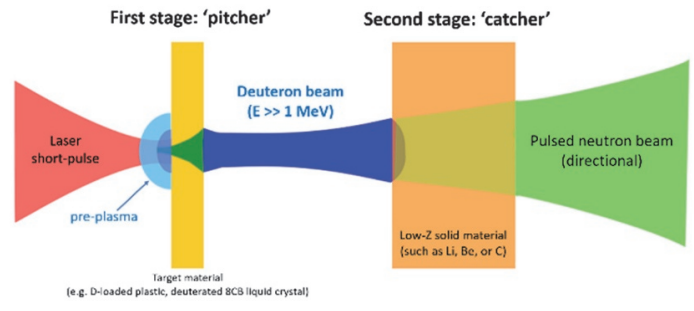

Fig. 1. Schematic of the principle of laser-driven neutron sources, see text for details.

Fig. 1 shows a schematic of a laser-driven neutron source using the ion-pitcher-catcher scenario. Two basic processes dominate: isotropic emission from nuclear reactions, where the incoming proton thermalizes its energy in the convertor and a neutron is emitted, and a directed component that generates neutrons while conserving the incoming ion momentum. The directed neutron generation component is dependent on two processes. The first is the so-called pre-equilibrium (or pre-compound) reaction, where the energy and momentum of the incoming proton or deuteron is directly transferred to a single nucleon in the convertor, resulting in the emission of an energetic neutron that conserves the initial momentum [11]. The second reaction is a stripping or breakup reaction. Here the incident deuterium is split or fissioned in the convertor and the resulting neutron conserves its initial momentum [12].

Results of our team and more detailed descriptions of the technique can be found in [5-7]. The reader is referred to these articles for more information.

\section{Qualitative Comparison with Conventional Pulsed Neutron Sources}

For compact neutron sources, $\mathrm{p}$ and $\mathrm{D}$ accelerator driven sources are well-developed. Such sources provide cost efficient access to thermal and fast neutrons, enabling applications in neutron radiography or neutron diffraction. The RANS source at the Riken accelerator complex [13] is a good example of such a source. While time-of-flight techniques were demonstrated for 
diffraction [13], the source, in pulsed mode, generates particle pulses that are at least $10 \mu$ s long. As shown in Fig. 2 , the moderation time for neutrons in the epithermal energy region $(\mathrm{E}>0.4 \mathrm{eV})$ is $1 \mu$ s or shorter. Therefore, this type of accelerator driven source cannot be used for energy-resolved epithermal neutron applications, e.g., to resolve nuclear resonances.

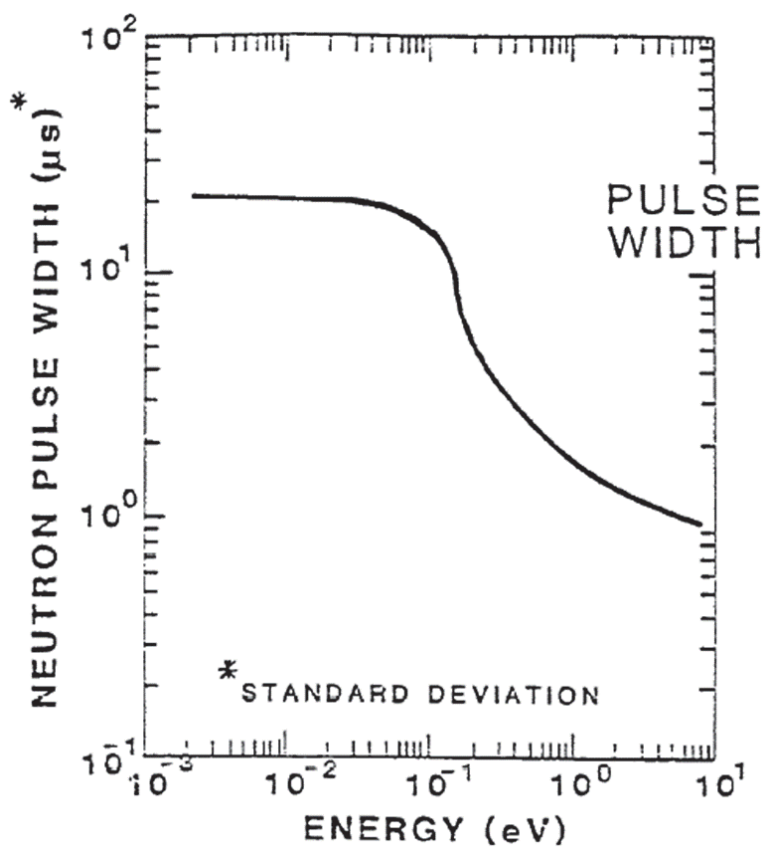

Fig. 2. Neutron pulse width emitted from a LANSCE thermal moderator as a function of neutron energy. While thermal neutron applications $(\mathrm{E}<0.4 \mathrm{eV})$ are possible with initial $\mathrm{p} / \mathrm{d}$ pulse widths of $\sim 10 \mu \mathrm{s}$, for epithermal applications pulses of this length would be much longer than the moderation time and therefore do not allow such applications (From: Gary Russell et al. ICANS-VIII Proceedings (1985) [14]).

The pulse structure in conventional RF accelerator-driven sources is strongly influenced by beam space-charge. Short pulse spallation sources typically use proton LINACS, coupled with storage rings, or proton synchrotrons to produce intense, sub-microsecond neutron pulses. There is, however, a limit on the number of protons or ions that can be stored in a conventional storage ring due to space-charge tune-depression [15]. Limits on the number of protons per pulse directly imply limits on the pulse density of neutrons. For example, the proton storage ring (PSR) at LANSCE compresses a 750 ms LINAC macro-pulse of $4 \times 10^{13}$ protons into a $270 \mathrm{~ns}$ pulse at $800 \mathrm{MeV}$ (the number of neutrons produced per proton by spallation in a tungsten target at $800 \mathrm{MeV}$ proton energy is approximately 20). Further reductions in compressed pulse length result in a concomitant reduction in protons per pulse, due principally to space charge effects. For example, pulse lengths of $1.5 \mathrm{~ns}, 5 \mathrm{~ns}$, and 10 $\mathrm{ns}$ in the LANSCE PSR were projected to store $1 \times 10^{11}$, $3 \times 10^{11}$, and $6 \times 10^{11}$ protons, respectively [16]. Finally, the cost for conventional accelerator pulse compressors or storage rings are significant and far exceed the investment cost for a compact neutron source such as RANS. Furthermore, space-charge limits the number of ions in a pulse, which constrains applications where high instantaneous flux with short temporal resolution is required, like in dynamic radiography or temperature measurements in dynamic events using neutron absorption resonance spectroscopy $[3,4]$.

Laser-driven neutron sources utilize photons to first accelerate electrons to high energy. The energetic electrons then couple to and accelerate ions by generating electrostatic fields due to charge separation. Charge separation, driven by the transport of energetic electrons, generates electric fields of $\mathrm{TV} / \mathrm{m}$. Ions are accelerated over typical distances of $\mathrm{L}_{\mathrm{ac}} \approx 10 \mu \mathrm{m}$ up to energies of approximately $94 \mathrm{MeV}$ for protons [17] and up to a few $\mathrm{MeV} / \mathrm{u}$ for heavier ions [18] using thin foil targets. Typical beam angular divergence, which is energydependent, is between $20^{\circ}-50^{\circ}$. The expansion of the ion beam results in an exponential energy distribution. The ion beam is highly directed with an emittance well below ion beams driven by conventional RF accelerators [1921].

In a conventional accelerator-driven source, significant effort is required to keep the ion pulses short and prevent the space-charge repulsion from spreading them in space. In contrast, in a laser-driven particle accelerator, the ions are accelerated in sub-picosecond timescales and propagate essentially with co-moving electrons. Thus space-charge driven beam divergence is small and beam emittance is preserved from the point of acceleration to the neutron-producing target. This fundamental difference between conventional accelerators and laser-driven particle accelerators allows lasers to achieve ultra-short pulse durations at intensities far greater than conventional accelerators. For example, at TRIDENT $10^{11}$ deuterons above $14 \mathrm{MeV}$ were produced in $500 \mathrm{fs}$ [5], equivalent to an instantaneous current of $10^{11}$ deuterons $\times 1.6 \cdot 10^{-19} \mathrm{C} / \mathrm{d} / 500 \mathrm{fs}=32 \mathrm{kA}$. Furthermore, there is a direct scaling between laser intensity and neutron pulse intensity. That is, more powerful lasers translate to higher neutron intensities per pulse depending on parameters such as the laser-matter interaction accelerating the deuterons or the energy-dependent crosssection of the deuteron/target interaction. The details of the relationship between laser power and neutron output are subject of ongoing research and therefore beyond the scope of this paper.

\section{Quantitative Comparison with the LANSCE Pulsed Sources}

This section provides a quantitative comparison between a pulse produced at the TRIDENT laser and at the LANSCE spallation source. 
At TRIDENT, $\sim 10^{10} \mathrm{n} /$ pulse were achieved during campaigns in March and July 2016, utilizing 70J output energy deposited in a 600 fs long pulse $(0.1 \mathrm{PW})$ of ultrahigh contrast [6]. This pulse produced deuterons with kinetic energies $\sim 20 \mathrm{MeV}$ interacting with the beryllium catcher target. Since the neutrons were pre-dominantly produced by the deuteron break-up reaction, the momentum of the incident deuteron was preserved and the produced neutrons travelled pre-dominantly in the direction of the laser pulse. Therefore, the majority of the neutrons were moderated by the polyethylene moderator in which the beryllium target was embedded (target/moderator coupling efficiency of $\sim 1$ ). For the sake of this estimation, we assume that all $\sim 10^{10}$ neutrons originating from a sub-nanosecond pre-moderation neutron pulse width were moderated (i.e. neglecting the small fraction of neutrons emitted into directions not covered by the moderator, see [5] for directional dependence of the neutrons emitted from the target).

LANSCE, on the other hand, is typically operating at $\sim 100 \mu \mathrm{A}$ proton current at $20 \mathrm{~Hz}$ with protons having an energy of $800 \mathrm{MeV}$. This converts to $\sim 3 \times 10^{13}$ protons per pulse hitting the tungsten spallation target. Assuming a conversion rate of 20 neutrons per proton, this results in $\sim 6 \times 10^{14}$ neutrons per pulse. However, these neutrons are emitted isotropically from the $\sim 10 \mathrm{~cm}$ diameter, $20 \mathrm{~cm}$ tall upper spallation target [22]. To avoid exposure of the beam lines with the intense $\gamma$ flash from the spallation pulse, the moderators serving the beam lines are below this target and above a lower target in a so-called split target configuration [22]. Estimating that 2\% [23] of the produced neutrons cross the moderator surface of one of the four moderators (because of the moderators viewing a void rather than target material), $\sim 1 \times 10^{13}$ moderated neutrons are emitted per proton pulse with a $\sim 270 \mathrm{~ns}$ proton pulse length.

A beam line viewing the moderator at TRIDENT therefore receives about three orders of magnitude less neutrons than a beamline viewing a LANSCE moderator. However, concepts to improve the deuteron flux over that produced by simple deuterated plastic targets as well as optimizations of the thermal and epithermal neutrons produced for a given application over that of the simple polyethylene block used as a moderator at TRIDENT are predicted to allow for a gain of about an order of magnitude. Furthermore, lasers providing higher energies than the $\sim 70 \mathrm{~J}$ provided by the TRIDENT system are available today, e.g. kJ systems (albeit not yet for repetition rates suitable for a pulsed neutron source). A higher laser energy would produce both more accelerated deuterons as well as a higher kinetic energy of the deuterons, translating to a gain in neutron pulse intensity. Laser systems such as the Scalable High-average-power Advanced Radiography Capability (SHARC) from Livermore National Lab provide 150J at $10 \mathrm{~Hz}$ [24]. Systems that are even more powerful and deliver laser pulses at $\sim 10 \mathrm{~Hz}$ are under development. However, no laser system was designed yet specifically as a driver for a laser-driven neutron source. The latter is likely to offer further improvements as current lasers for laser facilities are designed for a broad range of laser parameters, serving many applications ranging from plasma physics to laserdriven dynamic compression $[25,26]$.

It is worth pointing out that the cross-section of the deuteron break-up reaction increases neutron yield better than linear with deuteron energy, which correlates linearly with laser energy. In the foreseeable future, therefore, laser-driven neutron sources that are about an order of magnitude weaker than LANSCE appear feasible. For spallation sources the neutrons produced by the intranuclear cascade process reach energies up to the proton energy (e.g. $\sim 1 \mathrm{GeV}$ ) [27]. However, it is this small fraction of neutrons which drive the shielding demands. Modern spallation sources have their closest sample position outside the bulk shield at $\sim 15-20 \mathrm{~m}$, e.g. the high pressure beamline SNAP at the SNS has a moderator to sample distance of $\sim 15 \mathrm{~m}$ [28]. At LANSCE, the bulk shield ends at $4.5 \mathrm{~m}$ with the closest possible sample position around $6 \mathrm{~m}$. Laser-driven neutron sources, similar to the RANS source [13], require significantly less shielding of $\sim 1 \mathrm{~m}$ since neutrons are not produced by spallation. For some applications, such as epithermal energy-resolved neutron imaging, allowing to measure isotope densities [10], the resolution available with a shorter flight path is acceptable. The concurrent flux increase from the $1 / \mathrm{L}^{2}$ ratio of the LANSCE FP5/ERNI beamline [29], where these experiments are presently conducted at around $10 \mathrm{~m}$ sample to detector distance, would therefore enable some applications at neutron count times similar to what is required at LANSCE.

Compact neutron sources typically serve only a single or a few rather than several tens of beamlines as is the case for large-scale user facilities. All design studies and optimizations conducted by the UCANS or projects such as the Jülich high-brilliance neutron source project [1] for compact accelerator-driven neutron sources are immediately applicable for laser-driven neutron sources.

\section{Ongoing Efforts}

Concepts and efforts for laser deuteron catcher target systems were summarized in a report by our team [7], including a discussion of alternative solutions for the solid targets, left with a hole after each laser shot, such as gas or liquid cryo-jet target systems. Our team collaborates with laser facilities and will utilize them to produce neutrons as they become available after required upgrades, e.g. to improve laser contrast or enable higher repetition rates. In order to demonstrate the usability of these laser sources for applications, a multi-modality neutron instrument called "LANSCE-in-a-box" was designed and is in commissioning at the FP5 beam line at LANSCE. 


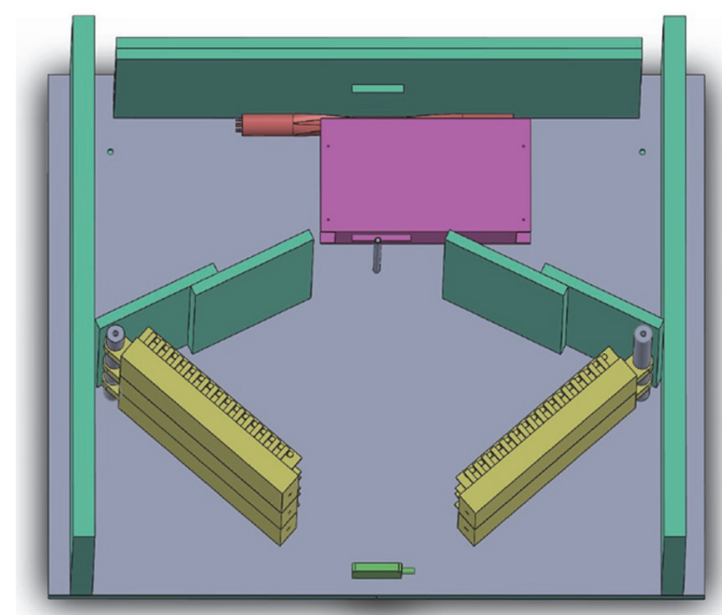

Fig. 3. Schematic of the LANSCE-in-a-box multi-modal portable neutron instrument. The dimensions of the square baseplate are $\sim 1 \mathrm{~m}$. From bottom to top, the detectors are a fission chamber (green), $96{ }^{3} \mathrm{He}$ tubes on three panels (yellow), a lightbox for scintillator-based imaging with a CMOS camera (magenta), and a dual-photomultiplier neutron transmission detector viewing a ${ }^{6} \mathrm{Li}$ glass scintillator. The sample is along the centreline of the instrument in front of the imaging lightbox. The green walls are borated polyethylene.

Fig. 3 shows a schematic of the setup. The instrument is designed to be portable and to require only $\sim 1 \mathrm{~m}^{2}$ in area to allow deploying in the target area of laser sources not necessarily designed for time-of-flight neutron instruments. Collimation and shielding will be part of the setup. After calibration at LANSCE, the diffraction detectors will only require the source to sample distance to be adapted at a different facility before the histograms from the $96{ }^{3} \mathrm{He}$ tubes can be integrated into a single histogram of high statistical quality, removing the requirement to calibrate sample-detector distances at a weak source. A sample such as an additively manufactured tungsten object, approximately of cylindrical shape, will allow to demonstrate simultaneous diffraction with the ${ }^{3} \mathrm{He}$ detectors, imaging with an imaging detector, and neutron resonance spectroscopy from the intense $18.6 \mathrm{eV}^{182} \mathrm{~W}$ resonance with the dualphotomultiplier coincidence setup viewing a ${ }^{6} \mathrm{Li}$ glass scintillator. Data collected at LANSCE with single, ten, one hundred and one thousand LANSCE neutron pulses will allow comparison of the per-pulse neutron intensity produced at a laser source. Fig. 4 shows a photograph of the first experiments with the setup in September 2019 at the FP5 beamline at LANSCE.

\section{Conclusions}

The promise of ultra-intense lasers as drivers for brilliant, compact, and highly efficient particle accelerators portends driving next-generation neutron sources, potentially replacing in some cases much larger conventional accelerators. For medium size neutron sources, as well as for high-peak-flux neutron sources, the

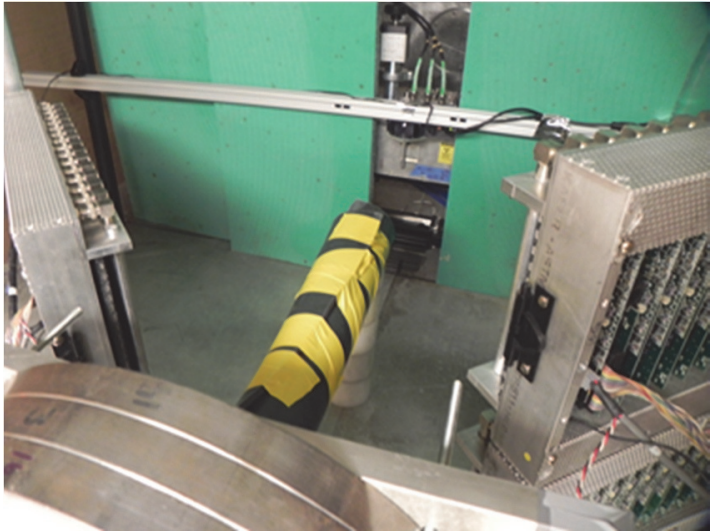

Fig. 4. Photograph of the first tests of the LANSCE-in-a-box multi-modal portable neutron instrument. The ${ }^{3} \mathrm{He}$ detector panels as well as the multi-channel plate imaging detector (behind green borated polytehtylene walls) are visible. A $6 \mathrm{~mm}$ diameter vanadium can with a sample is visible in front of the imaging detector. The black borated plastic, rolled into a pipe, reduced air scatter.

performance of laser sources is evolving to where they are both cost and performance competitive with conventional accelerators. Furthermore, laser sources are relatively compact (similar to the RANS source, i.e. several hundred $\mathrm{m}^{2}$ including power supplies and diagnostic systems, fitting in a modest lab) and can be easily attached to secondary drivers such as accelerators, other lasers, or pulsed power machines. The fundamental difference is that the acceleration potential for particle acceleration in short pulse lasers is $\mathrm{TV} / \mathrm{m}$ compared to the tens of $\mathrm{MV} / \mathrm{m}$ in conventional RF accelerators. Laser plug to beam efficiencies today are slightly below that of RF accelerators with rapid improvements occurring. The precedence of high-powered lasers in rugged environments for military or industrial (e.g. welding) applications may even provide a pathway to mobile compact short-pulse neutron sources. The Lyncean Compact Light Source [30], replacing an undulator with a laser to produce synchrotron X-rays in-house, may be transformative for the synchrotron community. The neutron community may experience a transformation enabled by lasers, too.

We wish to thank an anonymous reviewer for thoughtful comments greatly improving the manuscript. MR and NM acknowledge financial support for visits to LANSCE by the Institute for Materials Science at Los Alamos via an IMS Rapid Response grant. SCV acknowledges support by the DOE/NE Fuel Cycle R\&D research program. This work has benefitted from the use of the Los Alamos Neutron Science Center (LANSCE) at Los Alamos National Laboratory. Los Alamos National Laboratory is operated by Triad National Security, LLC, for the National Nuclear Security Administration of the U.S. Department of Energy under contract number 89233218 NCA000001. 


\section{References}

1. U. Rücker, T. Cronert, J. Voigt, J. P. Dabruck, P-E. Doege, J. Ulrich, R. Nabbi et al., European Physical Journal Plus 13119 (2016).

2. H. G.Priesmeyer, and U. Harz, Atomkernenergie 25.2 109-113 (1975).

3. V.W. Yuan, J.D. Bowman, D.J. Funk, G.L. Morgan, R.L. Rabie, C.E. Ragan, J.P. Quintana, and H.L. Stacy, Phys. Rev. Lett. 94125504 (2005).

4. J.C. Fernández, C.W. Barnes, M.J. Mocko, L. Zavorka, Rev. Sci. Instr. 90094901 (2019).

5. M. Roth et al., Phys. Rev. Lett. 110044802 (2013).

6. J.C. Fernández, D. Cort Gautier, C. Huang, S. Palaniyappan, B.J. Albright, W. Bang, G. Dyer, A. Favalli, J.F. Hunter, J. Mendez, S. C. Vogel, M. Roth et al., Phys. Plasmas, 24056702 (2017).

7. M. Roth, S.C. Vogel, M.A.M. Bourke, J.C. Fernandez, M.J. Mocko, S. Glenzer, W. Leemans, C. Siders, C. Haefner, Assessment of Laser-Driven Pulsed Neutron Sources for Poolside Neutron-based Advanced NDE-A Pathway to LANSCE-like Characterization at INL, Los Alamos National Lab. Los Alamos, NM, U.S.A., report No. LA-UR-1723190 (2016).

8. S.C. Vogel, M. Roth, J.C. Fernandez, D.C. Gautier, K.F. Schoenberg, Neutron News, 29 32-36 (2018).

9. A. Favalli, N. Guler, D. Henzlova, S. Croft, K. Falk, D.C. Gautier, K.D. Ianakiev, M. Iliev, S. Palaniyappan, M. Roth, and J.C. Fernandez, Scientific Reports, 92004 (2019).

10. A.S. Tremsin, S. C. Vogel, M. Mocko, M. A. M. Bourke, V. Yuan, R. O. Nelson, D. W. Brown, W. B. Feller, J. Nucl. Mat. 440 633-646 (2013).

11. G.D. Harp, J.M. Miller, Phys, Rev. C, 3(5), 1847 (1971).

12. Y. Watanabe, T. Ye, K. Ogata, In: EPJ Web of Conferences, 2, 11003 (2010).

13. Y. Otake, Plasma and Fusion Research $132401017-$ 2401017 (2018).

14. G.J. Russell, C. D. Bowman, E. R. Whitaker, H. Robinson, M. M. Meier. In: ICANS-VIII: Proceedings of the Eighth Meeting of the International Collaboration on Advanced Neutron Sources, Rutherford-Appleton Laboratory, Report RAL-85-110, pp. 272-293 (1985).

15. A.G. Ruggiero, AIP Conference Proceedings, 468 244-257 (1999).

16. R Garnett, Los Alamos National Laboratory, private communication (2019).

17. A. Higginson, R. J. Gray, M. King, R. J. Dance, S. D. R. Williamson, N. M. H. Butler, R. Wilson et al. Nature Comm. 9724 (2018).

18. M. Hegelich, S. Karsch, G. Pretzler, D. Habs, K. Witte, W. Guenther, M. Allen et al., PRL 89085002 (2002).
19. T.E. Cowan, J. Fuchs, H. Ruhl, A. Kemp, P. Audebert, M. Roth, R. Stephens et al., PRL 92 204801 (2004).

20. M. Schollmeier, M. Roth, A. Blazevic, E. Brambrink, J. A. Cobble, J. C. Fernandez, K. A. Flippo et al., NIMA 577 186-190 (2007).

21. J.A. Cobble, R. P. Johnson, T. E. Cowan, N. RenardLe Galloudec, M. Allen, J. Appl. Phys. 92 1775-1779 (2002).

22. M. Mocko, G. Muhrer, NIMA 704 27-35 (2013).

23. M. Mocko, personal communication.

24. E. Sistrunk, D. A. Alessi, A. Bayramian, K. Chesnut, A. Erlandson, T. C. Galvin, D. Gibson et al., In: Short-pulse High-energy Lasers and Ultrafast Optical Technologies, 11034, 1103407. International Society for Optics and Photonics (2019).

25. G. Mourou, T. Toshiki, Optics and Photonics News 22 47-51 (2011).

26. National Academies of Sciences, Engineering, and Medicine. Opportunities in Intense Ultrafast Lasers: Reaching for the Brightest Light. National Academies Press (,2018).

27. G.H. Russell, Proceedings CANS-XI : International Collaboration on Advanced Neutron Sources, KEK. Tsukuba. October 22-26,1990, 291-299 (1990).

28. A.S. Tremsin, J. B. McPhate, J. V. Vallerga, O. H. W. Siegmund, W. B. Feller, H. Z. Bilheux, J. J. Molaison et al., J. Phys.: Conference Series, 251012069 (2010).

29. R. Nelson, Ronald, S.C. Vogel, J.F. Hunter, E.B. Watkins, AS. Losko, As. Tremsin, N.P. Borges et al., J. Imaging 445 (2018).

30. E. Eggl, M. Dierolf, K. Achterhold, C. Jud, B. Günther, E. Braig, B. Gleich, F.Pfeiffer, J. Synchrotron Rad. 23 1137-1142 (2016). 